\title{
FULLY LEFT BOUNDED LEFT NOETHERIAN RINGS
}

John Beachy

A left Noetherian ring $R$ is fully left bounded $\rightleftarrows$ for each cyclic module $R^{M}$ there exist elements $m_{l}, \ldots, m_{n} \in M$ such that $\operatorname{Ann}(M)=\operatorname{Ann}\left(m_{1}, \ldots, m_{n}\right)$. Question: For what rings is there a uniform bound on the number of elements required? In particular, does a Noetherian ring with polynomial identity have a uniform bound? The condition is easily seen to be satisfied for any left Artinian ring or any ring finitely generated (as a module) over its center. A left Noetherian ring has bound one if and only if every left ideal is two-sided. 